\title{
THE ACCESS THEOREM FOR SUBHARMONIC FUNCTIONS
}

\author{
BY
}

\author{
R. HORNBLOWER AND E. S. THOMAS, JR.(1)
}

\begin{abstract}
A chain from a point $z_{0}$ of the open unit disk $\Delta$ to the boundary of $\Delta$ is a set $\Gamma=\bigcup\left\{\gamma_{n} \mid n=0,1,2, \ldots\right\}$ where the $\gamma_{n}$ are compact, connected subsets of $\Delta, z_{0}$ is in $\gamma_{0}, \gamma_{n}$ meets $\gamma_{n+1}$ and the $\gamma_{n}$ approach the boundary of $\Delta$. The following "Access Theorem" is proved: If $u$ is subharmonic in $\Delta, z_{0}$ is a point of $\Delta$ and $M<u\left(z_{0}\right)$, then there is a chain from $z_{0}$ to the boundary of $\Delta$ on which $u \geq M$ and on which $u$ tends to a limit. A refinement, in which the chain is a polygonal arc, is established, and an example is constructed to show that the theorem fails if $M=u\left(z_{0}\right)$ even for bounded, continuous subharmonic functions.
\end{abstract}

1. Introduction. In [5], the subharmonic analogues $\mathfrak{A}_{s}, \mathfrak{B}_{s}, \mathcal{C}_{s}$ of MacLane's classes $\mathscr{A}, \mathfrak{B}, \mathcal{L}[6]$, were introduced and studied. It was shown that these classes $\mathscr{C}_{s}, \mathfrak{B}_{s}, \mathcal{C}_{s}$ were identical; a key step in the proof was Theorem $2, \S 4$ of [5] which, because of its topological content, we have labeled the Access Theorem. This result is of some interest in itself since it sheds light on both the topological and analytical behavior of subharmonic functions.

The original proof of the theorem was lengthy and rather technical. In this paper, we shall give a shorter and more conceptual proof, a polygonal version of the theorem, and, perhaps of equal importance, an example which shows that the natural sharpening of the theorem is false.

To state our results precisely we introduce some notation and terminology. The open unit disk is denoted $\Delta$ and its boundary $\partial \Delta$. The word continuum means compact connected set.

Definition 1. If $z_{0}$ is a point of $\Delta$, a chain from $z_{0}$ to $\partial \Delta$ is a subset $\Gamma$ of $\Delta$ which can be written as the union of a sequence of continua $\left\{\gamma_{n} \mid n=\right.$ $0,1,2, \ldots\}$ in $\Delta$ with the properties that $z_{0} \in \gamma_{0}, \gamma_{n} \cap \gamma_{n+1} \neq \varnothing$ for all $n$, and, for any compact set $E$ in $\Delta$, only finitely many $\gamma_{n}$ meet $E$. Thus, a chain is a connected set consisting of a sequence of links, $\gamma_{n}$, which tend toward $\partial \Delta$ and the first of which contains $z_{0}$.

Let $\mu$ be a function defined in $\Delta$ with values in the extended reals and let $\Gamma=\bigcup \gamma_{n}$ be a chain from $z_{0} \in \Delta$ to $\partial \Delta$.

Received by the editors March 9, 1971. 30A90.

AMS (MOS) subject classifications (1970). Primary 31A05, 31A20; Secondary 30A72,

Key words and phrases. Subharmonic function, harmonic function, MacLane classes, Milloux-Schmidt inequality, conformal map.

(1) Research partially supported by National Science Foundation Grant GU 31.71. 
Definition 2. We say that $u$ tends to a limit, $L$, on $\Gamma$ provided $\lim M_{n}=$ $\lim m_{n}=L$ where $M_{n}=\max _{z \in \gamma_{n}} u(z), m_{n}=\min _{z \epsilon \gamma_{n}} u(z)$ and limits are taken in the extended sense.

We are now in a position to state the Access Theorem.

Theorem A. Let $u$ be a subbarmonic function defined in $\Delta$ and let $M<u\left(z_{0}\right)$ where $z_{0}$ is a point of $\Delta$. There is a chain $\Gamma$ from $z_{0}$ to $\partial \Delta$ on which $u \geq M$ and on which $u$ tends to a limit.

The proof of this theorem and a refinement, Theorem $B$, in which the links of the chain are polygonal arcs, will be given in $\$ 2$; we remark that the result is easy to prove for harmonic functions using a modification of an argument of $G$. $R$. MacLane [6, p. 7]. In this case one can replace the condition $M<u\left(z_{0}\right)$ by $M \leq$ $u\left(z_{0}\right)$; it is natural to ask whether this is true in general.

In $\$ 3$ we show that the answer is no by constructing a bounded, continuous, subharmonic function $w$ in $\Delta$ such that the set where $w \geq w(0)$ does not even contain a chain from zero to $\partial \Delta$.

In the remainder of this section we list some properties of subharmonic functions. For details, the reader is referred to [3].

To begin, a function $u$ is subharmonic in an open subset $W$ of the complex plane provided the following three conditions are satisfied:

(i) $u: W \rightarrow[-\infty, \infty)$,

(ii) $u$ is upper semicontinuous (u.s.c.) in $W$, and

(iii) for all small enough $r, u$ satisfies the mean value inequality

$$
u(z) \leq \frac{1}{2 \pi} \int_{0}^{2 \pi} u\left(z+r e^{i \theta}\right) d \theta \quad \text { for all } z \in W .
$$

where the integral is in the Lebesgue sense.

A function $u$ satisfying (i) and (ii) is subharmonic in $W$ if and only if the following condition holds:

(iii) ${ }^{\prime}$ If $v$ is a harmonic function defined in an open subset $W^{\prime}$ of $W$, then $u-v$ is either constant or fails to have a maximum in $W^{\prime}$.

Condition (iii)' yields the following maximum principle: if $u$ is subharmonic in an open set $W$ and $u$ is not constant, then for each $z$ in $W, u(z)<\max _{z \in W} u(z)$.

In the proof of the Access Theorem we shall need the following fact which is a consequence of Brelot's form of the Milloux-Schmidt inequality (see [1]) and the mean value inequality, (iii), given above.

Lemma. If $u$ is subbarmonic and bounded above in $W$ and if $z \in W$, then, for any $\epsilon>0$, there are circles of arbitrarily small radius centered at $z$ on which $u \geq u\left(z_{0}\right)-\epsilon$. 
2. The theorem. The proof of the theorem involves an inductive construction, each step of which uses two lemmas which we prove first. We need a preliminary definition.

Definition 3. Let $u$ be a function defined in $\Delta$, let $z_{0}$ be a point of $\Delta$ and let $M \leq u\left(z_{0}\right)$. We say that a point $z$ of $\Delta$ belongs to the compartment of $z$ relative to $M$ provided there is a continuum in $\Delta$ containing both $z_{0}$ and $z$ on which $u \geq M$. The compartment of $z_{0}$ relative to $M$ is denoted $C\left(z_{0}, M\right)$.

Remarks. This definition was given in [5]. $C\left(z_{0}, M\right)$ need not be closed in $\Delta$ and, in particular, $C\left(z_{0}, M\right)$ does not necessarily coincide with the component of $z_{0}$ in $\{u \geq M\}$, as the example in $\$ 3$ shows.

Lemma 1. If $u$ is subbarmonic in $\Delta$ and $z_{0}$ is a point of $\Delta$ then $C\left(z_{0}, u\left(z_{0}\right)\right)$ bas limit points in $\partial \Delta$.

Proof. Fix $R$, with $\left|z_{0}\right|<R<1$ and let $K=\{|z| \leq R\} \cap\left\{u \geq u\left(z_{0}\right)\right\}$. The set $K$ is compact and we assert that $Q=K \cup\{|z|=R\}$ is connected. If not, then we may write $Q=A \cup B$ where $A, B$ are compact, disjoint, nonempty and $\{|z|=R\}$ lies in $B$.

Clearly there is an open subset $W$ of $\{|z| \leq R\}$ such that $A \subset W$ and the closure of $W$ misses $B$. Then, in fact, $W$ is open in $\Delta$ and its boundary lies in the set $\{u<M\}$. But then $\max _{z \in W} u(z)$ must be achieved in $A \subset W$ which contradicts the maximum principle for subharmonic functions.

Therefore, $Q$ is connected. This implies that there is a continuum in $\Delta$ containing $z_{0}$ and meeting $|z|=R$ (namely, the component of $Q$ which contains $z_{0}$ ). Since $R$ may be chosen arbitrarily close to one this proves the lemma.

Lemma 2. Let $u$ be a nonconstant subbarmonic function in $\Delta, z_{0}$ a point of $\Delta$ and let $M<u\left(z_{0}\right)$. Then

(a) $\max u \mid C\left(z_{0}, M\right)>u\left(z_{0}\right)$, and

(b) if $\left|z_{0}\right|<R<1$ and if $C(R)=C\left(z_{0}, M\right) \cap\{|z| \leq R\}$, then $\max u \mid C\left(z_{0}, M\right)>$ $\max u \mid C(R)$.

Remark. Although certain steps in the proof have been stated as lemmas in [7] and [5] we shall, for completeness, fill in all details.

Proof of (a). Given $R$, with $\left|z_{0}\right|<R<1$, define an auxiliary function $v$ on the set $|z| \leq R$ by

$$
v(z)= \begin{cases}u(z) & \text { if } z \in C\left(z_{0}, M\right), \\ M & \text { otherwise. }\end{cases}
$$

We show that $v$ is subharmonic in $|z|<R$.

Certainly $v$ satisfies property (i), so we need to verify that properties (ii) and 
(iii) hold at each point of $\{|z|<R\}$. We need only consider points which lie in the boundary of $C\left(z_{0}, M\right)$ relative to $\{|z|<R\}$, since at other points $v$ is either locally constant or locally equal to $u$.

Fix such a point $z_{1}$ and suppose first that $u\left(z_{1}\right)=M$, whence $v\left(z_{1}\right)=M$. Then certainly $v$ satisfies the mean value inequality, (iii), at $z_{1}$ since $v \geq M$ every where in $\{|z|<R\}$. Given $\epsilon>0$, there is a neighborhood $U$ of $z_{1}$ on which $u \leq(M+\epsilon)$. On this same neighborhood we also have $v \leq(M+\epsilon)$. Since $\epsilon$ is arbitrarily small, this shows that $v$ is upper semicontinuous at $z_{1}$.

Secondly, suppose $u\left(z_{1}\right)>M$. Choose $\epsilon>0$ so that $\left(u\left(z_{1}\right)-\epsilon\right)>M$. By Brelot's form of the Milloux-Schmidt inequality there is a circle $\sigma$ centered at $z_{1}$, and lying in $\{|z|<R\}$ on which $u \geq\left(u\left(z_{1}\right)-\epsilon\right)$. Since $z_{1}$ is a limit point of $C\left(z_{0}, M\right), C\left(z_{0}, M\right)$ must meet $\sigma$ and this implies that $\sigma \subset C\left(z_{0}, M\right)$. From Lemma 1 it then follows that if $z$ lies inside $\sigma$ and $u(z) \geq M$ then $z \in C\left(z_{0}, M\right)$. In particular, $z_{1} \in C\left(z_{0}, M\right)$ so that $v\left(z_{1}\right)=u\left(z_{1}\right)$. In addition, the definition of $v$ implies that, inside $\sigma$, we have $v \geq u$. It is then immediate that $v$ satisfies properties (ii) and (iii) at $z_{1}$.

This completes the verification that $v$ is subharmonic. Now suppose that $\max _{z \in C\left(z_{0}, M\right)} u(z)=u\left(z_{0}\right)$. Then $v(z) \leq u\left(z_{0}\right)$ for all $z$ in $|z| \leq R$ and, since $v\left(z_{0}\right)=u\left(z_{0}\right)$ it follows from the maximum principle that $v$ is constant, $v \equiv u\left(z_{0}\right)$, on $\{|z|<R\}$. This, in turn, implies that $u$ is constant on $\{|z|<R\}$. Since $R$ may be chosen arbitrarily close to one this contradicts our hypothesis that $u$ is nonconstant on $\Delta$. Thus, we must have $\max _{z \in C\left(z_{0}, M\right)} u(z)>u\left(z_{0}\right)$.

Proof of (b). Suppose instead that $\max u\left|C\left(z_{0}, M\right)=\max u\right| C(R)$ for some $R$ with $\left|z_{0}\right|<R<1$. Since $u$ is upper semicontinuous, there is a point $z_{1}$ in the closure of $C(R)$ with $u\left(z_{1}\right) \geq \max u \mid C\left(z_{0}, M\right)>u\left(z_{0}\right)$. Using the modified MillouxSchmidt inequality as we did above, we find that $z_{1} \in C\left(z_{0}, M\right)$. Applying (a) to $C\left(z_{1}, u\left(z_{1}\right)\right)$ we see that $\max u\left|C\left(z_{1}, u\left(z_{1}\right)\right)>u\left(z_{1}\right) \geq \max u\right| C\left(z_{0}, M\right)$. But this is a contradiction since $C\left(z_{1}, u\left(z_{1}\right)\right) \subset C\left(z_{0}, M\right)$. This completes the proof of (b).

Proof of the Access Theorem (Theorem A of the Introduction). The theorem is trivial for constant functions, therefore, let $u$ be a nonconstant subharmonic function in $\Delta$, let $z_{0}$ be a point of $\Delta$ and let $M<u\left(z_{n}\right)$. We must construct a chain $\Gamma$ from $z_{0}$ to $\partial \Delta$ lying in $C\left(z_{0}, M\right)$ on which $u$ tends to a limit.

We shall first deal with the case in which $u$ is bounded above on $C\left(z_{0}, M\right)$. The construction can be easily modified to cover the unbounded case and will be described afterwards.

To construct $\Gamma$ we produce a sequence of compartments $A_{n}=C\left(z_{n}, p_{n}\right)$ $(n=0,1,2, \ldots)$ and a sequence of continua $\gamma_{n}$, with the following properties:

1. $A_{0}=C\left(z_{0}, M\right)$,

2. the $A_{n}$ form a nested decreasing sequence, i.e. $A_{0} \supset A_{1} \supset A_{2} \supset \ldots$, 
3. the $A_{n}$ approach $\partial \Delta$, i.e. there is a sequence $R_{n}$ increasing to one such that $A_{n+1}$ lies in $\left\{|z| \geq R_{n}\right\}$,

4. $\gamma_{n}$ lies in $A_{n}, \gamma_{0}$ contains $z_{0}$ and $\gamma_{n} \cap \gamma_{n+1} \neq \varnothing$ for all $n$; and, finally,

5. for each $n \geq 1$ and each $z$ in $\gamma_{n}$ we have

$$
m_{n-1}-1 / 2^{n-1} \leq u(z) \leq m_{n} \text { where } m_{k}=\max u \mid A_{k} \text {. }
$$

(For $n=0$, we will have $M \leq u(z) \leq m_{0}$.)

Once these sets have been obtained we let $\Gamma=\bigcup \gamma_{n}$. By properties 1-4, $\Gamma$ is a chain from $z_{0}$ to $\partial \Delta$. By property 2 the $m_{n}$ form a nonincreasing sequence (bounded below by $M$ ) whose limit we denote by $L$. By the inequalities of condition $5, u$ tends to $L$ on $\Gamma$.

We now show how to carry out the construction.

Let $R_{0}$ be chosen so that $1 / 2<R_{0}$ and $\left|z_{0}\right|<R_{0}<1$, let $A_{0}=C\left(z_{0}, M\right)$, $B_{0}=A_{0} \cap\left\{|z| \leq R_{0}\right\}$ and let $m_{0}=\max u \mid A_{0}$ and $n_{0}=\max u \mid B_{0} \cdot$ By Lemma 2 (a), $m_{0}>n_{0}$ and therefore we can select a point $z_{1}$ in $A_{0}$ such that $u\left(z_{1}\right)=$ $\left(m_{0}-\epsilon_{0}\right)$ where $\epsilon_{0}$ satisfies $\epsilon_{0}<1 / 2$ and $\left(m_{0}-2 \epsilon_{0}\right)>n_{0}$. Let $\gamma_{0}$ be a continuum in $A_{0}$ containing $z_{0}$ and $z_{1}$, and, finally, let $A_{1}=C\left(z_{1}, p_{1}\right)$ where $p_{1}=\left(m_{0}-2 \epsilon\right)$. Figure 1 shows $A_{0}, \gamma_{0}$ and $A_{1}$.

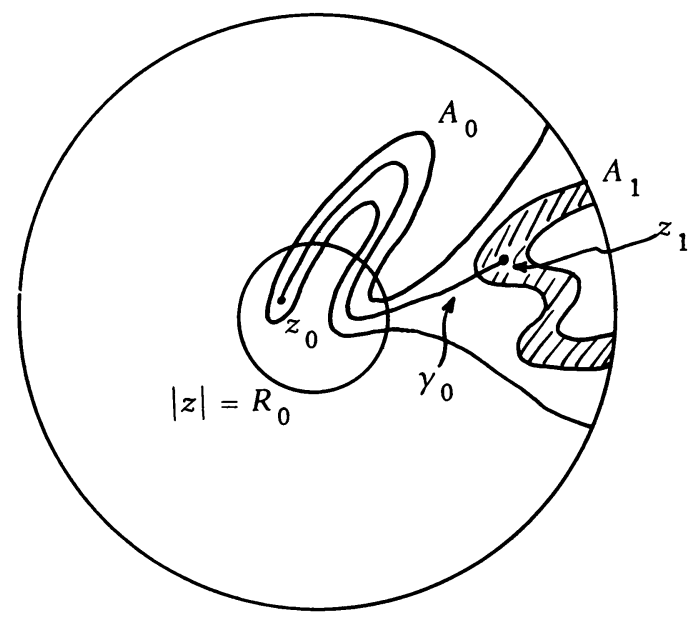

Figure 1

We now verify properties $1-5$. Note that $A_{0}$ contains $A_{1}$ because $z_{1} \in A_{0}$ and $p_{1}>n_{0} \geq M$. Also since $n_{0}=\max u \mid B_{0}$ and since $p_{1}>n_{0}$, the set $A_{1}$ must lie outside the circle $|z|=R_{0}$, by (b) of Lemma 2. Thus properties 2 and 3 have been checked, while properties 1,4 and 5 are immediate for $\gamma_{0}$ and $A_{0}$.

For the next step, we choose a number $R_{1}$ such that $3 / 4<R_{1}$ and $\gamma_{0}$ lies in 
$|z|<R_{1}$. Let $B_{1}=A_{1} \cap\left\{|z| \leq R_{1}\right\}$ and let $m_{1}=\max u \mid A_{1}$ and $n_{1}=\max u \mid B_{1}$. By Lemma 2 (a), $m_{1}>n_{1}$ and therefore we can select a point $z_{2}$ in $A_{1}$ such that $u\left(z_{2}\right)=\left(m_{1}-\epsilon_{1}\right)$ where $\epsilon_{1}$ satisfies. $\epsilon_{1}<1 / 4$ and $\left(m_{1}-2 \epsilon_{1}\right)>n_{1}$. Let $\gamma_{1}$ be a continuum in $A_{1}$ containing $z_{1}$ and $z_{2}$ and, finally, let $A_{2}=C\left(z_{2}, p_{2}\right)$ where $p_{2}=\left(m_{1}-2 \epsilon_{1}\right)$. Figure 2 illustrates the situation at this point.

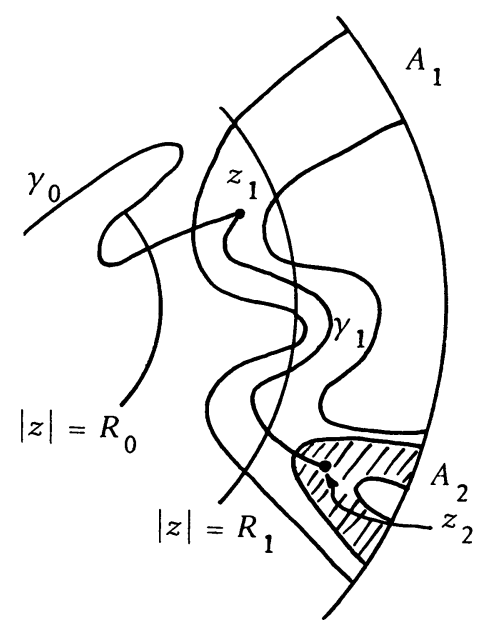

Figure 2

To verify properties $1-5$, note that as before $A_{2} \subset A_{1}$ since $z_{2} \in A_{2}$ and since $p_{2}>n_{1}=\max u \mid B_{1} \geq u(z)>p_{1}$. Also since $p_{2}>n_{1}, A_{2}$ lies outside the circle $|z|=R_{1}$ by Lemma $2(\mathrm{~b})$. Thus properties 2 and 3 hold for $A_{1}$. Since $z_{1}$ belongs to both $\gamma_{0}$ and $\gamma_{1}$ and since $\gamma_{1}$ was chosen to lie in $A_{1}$, property 4 holds. Finally, if $z_{1} \in \gamma_{1}$ then since $\gamma_{1} \subset A_{1}$ we have $u(z) \geq p_{1}=\left(m_{0}-2 \epsilon_{0}\right)>\left(m_{0}-1\right)$ and, trivially, $u(z) \leq m_{1}$. Thus 5 holds for $n=1$.

The remaining steps are carried out just as above with increased subscripts. The only delicate part of the construction is to make sure that at the $k$ th step $z_{k}$ is chosen so that $u\left(z_{k+1}\right)=\left(m_{k}-\epsilon_{k}\right)$ where $\epsilon_{k}$ satisfies $\epsilon_{k}<1 / 2^{k+1}$ and $\left(m_{k}-2 \epsilon_{k}\right)>n_{k}$. This insures that $A_{k+1} \subset A_{k}$ and $A_{k+1}$ lies outside $|z|=R_{k}$ and it guarantees that for any $z$ in $\gamma_{k+1}$ one will have

$$
u(z) \geq p_{k+1}=\left(m_{k}-2 \epsilon_{k}\right)>\left(m_{k}-1 / 2^{k}\right) .
$$

This finishes the proof in the case where $u$ is bounded on $C\left(z_{0}, M\right)=A_{0}$. If, instead, $\max u \mid A_{0}=+\infty$, then we choose $R_{0}, A_{0}, B_{0}, m_{0}=+\infty$ and $n_{0}=$ $\max u \mid B_{0}$ as before. Now we must have $n_{0}<+\infty$, since $u$ is upper semicontinuous and does not assume the value $+\infty$ on $\Delta$. Thus we can pick $z_{1}$ in $A_{0}$ such that $u\left(z_{1}\right) \geq\left(n_{0}+2\right)$. Let $\gamma_{0}$ be a continuum in $A_{0}$ containing $z_{0}$ and $z_{1}$ and let $A_{1}=C\left(z_{1}, p_{1}\right)$ where $p_{1}=\left(n_{0}+1\right)$. 
Properties 1-4 hold, as they did in the bounded case.

We next choose $R_{1}, B_{1}, m_{1}=\max u \mid A_{1}, n_{1}=\max _{z \in A_{1}} u(z)=\max _{z \in B_{1}} u(z)$ as before. If $m_{1}<+\infty$ then we proceed from here as in the bounded case. If $m_{1}=+\infty$, then $n_{1}<+\infty$ and we choose $z_{2}$ in $A_{1}$, such that $u\left(z_{2}\right) \geq$ $n_{1}+2$. Let $\gamma_{1}$ be a continuum in $A_{1}$ containing $z_{1}$ and $z_{2}$ and let $A_{2}=$ $C\left(z_{2}, p_{2}\right)$ where $p_{1}=\left(n_{1}+1\right)$.

Continue the construction in this way. If at some step $\max _{z \in A_{k}} u(z)<+\infty$, then proceed as in the bounded case. If at every step $\max _{z \in A_{k}} u(z)=+\infty$, then choose $z_{k+1}$ in $A_{k}$ such that $u\left(z_{k+1}\right) \geq\left(n_{k}+2\right)$, let $\gamma_{k}$ be a continuum in $A_{k}$ from $z_{k}$ to $z_{k+1}$ and let $A_{k+1}=C\left(z_{k+1}, p_{k+1}\right)$ where $p_{k+1}=\left(n_{k}+1\right)$. In this case the behavior $u$ on $\Gamma=\bigcup \gamma_{n}$ can be determined as follows. For $z$ in $\gamma_{k+2}$ we have $u(z) \geq p_{k+z}=\left(n_{k+1}+1\right)$ and $n_{k+1}=\max _{z \in B_{k+1}} u(z) \geq u\left(z_{1}\right)=\left(n_{k}+2\right)$. Thus, for $z$ in $\gamma_{k+2}$, we have $u(z) \geq\left(n_{k}+3\right) \geq\left(n_{0}+2 k+3\right)$, and therefore $u$ tends to $+\infty$ on $\Gamma$.

This completes the proof of Theorem A.

Remark. This proof does not go through in higher dimensions, although at least one of us thinks the theorem still holds. The problem is to find an analogue of Lemma 2, which guarantees that one can force nonconstant functions to increase as $z$ moves towards the boundary.

The chain obtained in the proof of this theorem need not be "nice;" indeed, the links $\left\{\gamma_{n}\right\}$ may be complicated continua and they may intersect each other in a messy fashion. Such pathology can be completely eliminated. Let us call a subset $A$ of $\Delta$ a polygonal arc from $z_{0}$ to $\partial \Delta$ if there is a homeomorphism $\phi$ of $[0,1)$ onto $A$ such that $\phi(0)=z_{0},|\phi(x)|$ approaches 1 as $x$ approaches 1 and $A$ is polygonal, i.e. $A$ is the union of a collection (necessarily infinite) of straight line segments.

Clearly, a polygonal arc can be regarded as a chain (in many ways) with polygonal arcs as links; moreover these arcs meet "nicely." If $A=\phi([0,1))$ is a polygonal arc from $z_{0}$ to $\partial \Delta$ then we say a function $u$ defined in $\Delta$ tends to a limit on $A$ if $u(\phi(x))$ tends to a limit (in the extended sense) as $x$ approaches 1. This, of course, coincides with the corresponding definition for arbitrary chains.

Theorem B. Let $u, z_{0}$ and $M$ be as in Theorem A. There is a polygonal arc A from $z_{0}$ to $\partial \Delta$ on which $u \geq M$ and on which $u$ tends to a limit.

Aside from a simple topology argument, the proof involves a lemma of Talpur which we state as follows:

Lemma $[7, \S 1]$. Suppose $u$ is subbarmonic in a neighborbood $U$ of a continuum $\gamma$ and that $u \geq M$ on $\gamma$. Given two points $w_{1}$ and $w_{2}$ of $\gamma$ and an $L<M$, there is a polygonal arc with endpoints $w_{1}$ and $w_{2}$, lying in $U$, on which $u \geq L$. 
We shall omit the proof of the lemma, except to point out that the polygonal arc can be obtained by piecing together short polygonal arcs whose existence is guaranteed by a deep result of Hayman [2, Theorem 4, p. 193].

Now to prove Theorem $B$, suppose $u, z_{0}$ and $M<u\left(z_{0}\right)$ are given. Let $M^{\prime}=$ $1 / 2\left(M+u\left(z_{0}\right)\right)$ and, using Theorem $A$, select a chain $\Gamma=\bigcup_{\gamma_{n}}$ from $z_{0}$ to $\partial \Delta$ on which $u \geq M^{\prime}$ and on which $u$ tends to a limit.

Let $p_{n}$ and $q_{n}$ denote the minimum and maximum of $u$ on $\gamma_{n}, n \geq 0$, and note that $p_{n} \geq M^{\prime}$ for all $n$ and that $q_{n}<+\infty$ for all $n$. For $n \geq 1$, let $z_{n}$ be a point of $\gamma_{n} \cap \gamma_{n+1}$. For $n \geq 0$, choose an open neighborhood $U_{n}$ of $\gamma_{n}$ lying in $\Delta$ such that:

(1) $U_{n}$ lies in the $1 / 2^{n}$ neighborhood of $\gamma_{n}$, and

(2) the maximum of $u$ on $U_{n}$ is at most $\left(q_{n}+1 / 2^{n}\right)$.

(The existence of such neighborhoods is guaranteed by the upper semicontinuity of u.)

Now we apply Talpur's lemma. For each $n \geq 0$, choose a polygonal arc $\beta_{n}$ lying in $U_{n}$ with endpoints $z_{n}$ and $z_{n+1}$ on which the following inequalities hold:

(3) If $z$ is in $\beta_{0}, u(z) \geq M$, and if $z$ is in $\beta_{n}, n \geq 1, u(z) \geq p_{n}-\epsilon_{n}$, where the $\epsilon_{n}$ are chosen so that $p_{n}-\epsilon_{n} \geq M$ and $\epsilon_{n} \rightarrow 0$.

The set $B=\bigcup \beta_{n}$ forms a chain from $z_{0}$ to $\partial \Delta$ (because of condition (1)) and on this chain $u \geq M$ and $u$ tends to the same limit as it did on the original chain (by (2) and (3)).

Now consider the set $(B \cup \partial \Delta)$, a closed connected subset of the closed disk $(\Delta \cup \partial \Delta)$. Collapse $\partial \Delta$ to a point; the quotient space is homeomorphic to $S^{2}$. Denoting the quotient map by $\pi, \pi:(\Delta \cup \partial \Delta) \rightarrow S^{2}$, we have that $\pi(\partial \Delta)$ is a point $p$ of $S^{2}$ and that $\pi \mid \Delta$ is a homeomorphism of $\Delta$ onto $S^{2}-p$.

The image of $B \cup \partial \Delta$ under $\pi$ is a compact connected set $C$ containing $\pi\left(z_{0}\right)$ and $p$. Moreover, because of the way $B$ was constructed, it is easy to see that $C$ is locally connected. Thus $C$ is a Peano continuum [4] and, as is well known, $C$ contains an arc with endpoints $\pi\left(z_{0}\right)$ and $p$. To be specific let $\psi$ be a homeomorphism of $[0,1]$ into $C$ such that $\psi(0)=\pi\left(z_{0}\right)$ and $\psi(1)=p$.

Now let $\phi:[0,1] \rightarrow \Delta$ be defined by $\phi(x)=\pi^{-1}(\psi(x))$. Then $\phi$ is a homeomorphism of $[0,1), \phi(0)=z_{0}$ and $|\phi(x)| \rightarrow \partial \Delta$ as $x \rightarrow 1$ (because $\pi(\phi(x)) \rightarrow p$ as $x \rightarrow 1)$. Moreover $\phi([0,1))$ lies in $B$ so that it must be polygonal. Finally, it is clear that on this polygonal arc, $u$ tends to the same limit as it did on $B$.

Remark. This proof also fails in higher dimensions. The problem here is that the result of Hayman no longer holds and the key result of Talpur is not established.

3. The example. In this section we shall construct a bounded, continuous function $w$, subharmonic in $\Delta$, taking on the value 0 at the origin $z_{0}$, but such that $C\left(z_{0}, 0\right)$ contains no chain from $z_{0}$ to $\partial \Delta$. 
Let $S$ be a copy of the real line which is smoothly embedded in $\Delta$ so that $z_{0}$ is a point of $S$ and the closure of $S$ consists of $S$ and two disjoint vertical segments spanning $\Delta$. For definiteness, let us take

$$
S=\left\{(x, y) \in \Delta \mid-\frac{3}{4} \leq x \leq \frac{3}{4} \text { and } y=\frac{\sqrt{7}}{4} \sin g(x) \text { where } g(x)=\frac{16}{9-16 x^{2}}-\frac{16}{9}\right\} \text {. }
$$

Then the closure of $S$ in $\Delta$ consists of $S$ and the segments $\{( \pm(3 / 4), y) \mid-\sqrt{7} / 4$ $<y<\sqrt{7} / 4\}$ (see Figure 3).

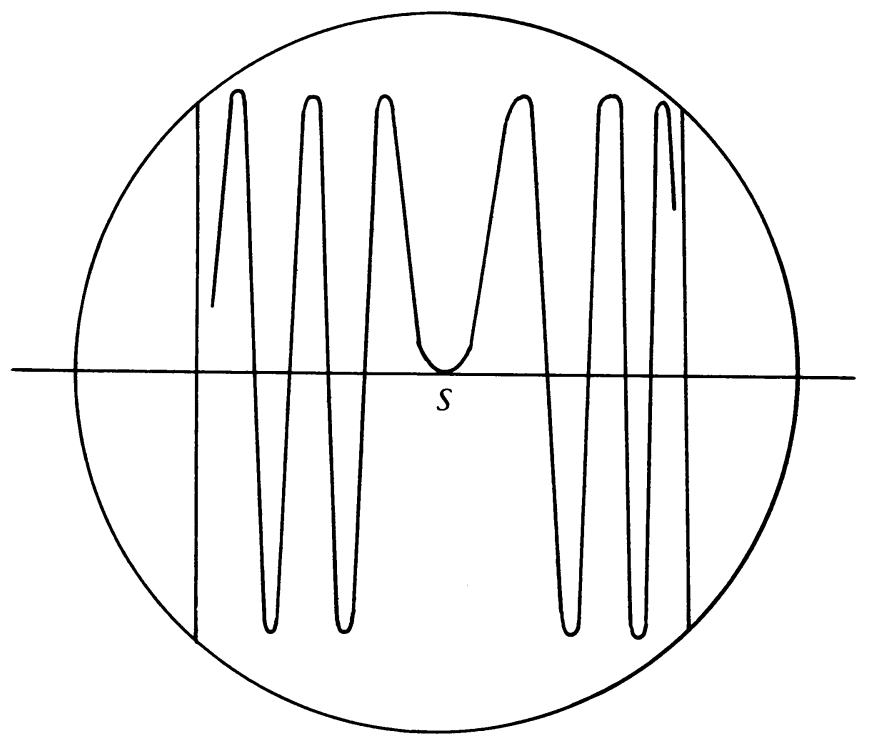

Figure 3

Since $S$ is smoothly embedded in $\Delta$ we can choose a sequence $\left\{U_{n}\right\}$ of wavy open strips with disjoint closures which lie above $S$, between the lines $x=$ $\pm(3 / 4)$ and which converge monotonically to $S$. Figure 4 , in which the oscillations of $S$ have been flattened, illustrates the desired configuration.

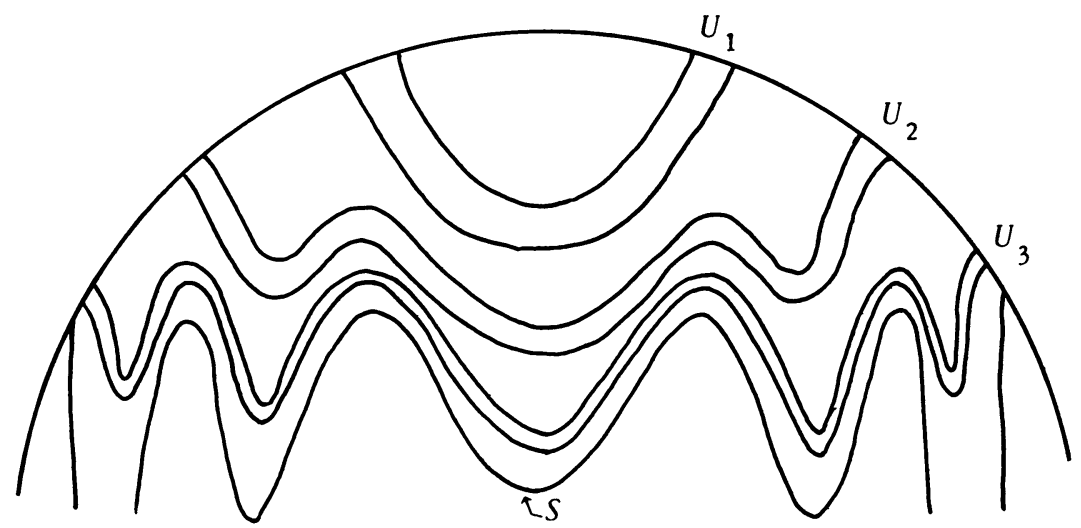

Figure 4 
The boundary of each wavy strip consists of four arcs; the two arcs which lie in $\Delta$ except for the endpoints will be called the top and bottom edges and the two lying in $\partial \Delta$ will be called the left and right edges. Each strip $U_{n}$ is to be chosen so that there is a conformal map $\phi_{n}$ defined in a neighborhood of the closure $\bar{U}_{n}$ of $U_{n}$ which takes $\bar{U}_{n}$ homeomorphically onto a closed rectangle $R_{n}=\{(x, y) \mid$ $\left.-K_{n} \leq x \leq K_{n}, 0 \leq y \leq 3 \pi\right\}$ with the edges of $\bar{U}_{n}$ going onto the corresponding edges of $R_{n}$. (Observe that since the $U_{n}$ 's must have "width" tending to 0 and "length" tending to $+\infty$, the numbers $K_{n}$ will tend rapidly to $+\infty$.)

Let $v_{n}$ be defined on $R_{n}$ by $v_{n}(z)=\operatorname{Im}\left(M_{n} e^{z}\right)$ where $M_{n}=e^{-K_{n} / n}$. Thus $v_{n}$, being the imaginary part of a holomorphic function, is harmonic (and a fortiori subharmonic) in the interior of $R_{n}$. Moreover $\left|v_{n}\right|<1 / n$ on $R_{n}, v_{n}$ has the value zero on the top and bottom edges and has negative values in the subrectangle of $R_{n}$ given by $\pi<y<2 \pi$. Qualitatively, the graph of $v_{n}$ is a wave form of amplitude at most $1 / n$ having two crests and one trough.

We now pull this wave back to $\bar{U}_{n}$; for $z$ in $\bar{U}_{n}$ let $u_{n}(z)=v_{n}\left(\phi_{n}(z)\right)$. As is well known [3], the composition of a subharmonic function and a holomorphic function is subharmonic, thus each $u_{n}$ is subharmonic on $U_{n}$, continuous on $\bar{U}_{n}$, vanishes on the top and bottom edges of $\bar{U}_{n}$ and $\left|u_{n}\right| \leq 1 / n$ on $\bar{U}_{n}$.

In a similar manner we may construct a sequence of strips $\left\{U_{n}^{\prime}\right\}$ converging to $S$ from below and on each of these a subharmonic wave form $u_{n}^{\prime}$ satisfying properties analogous to the $u_{n}$.

We now define a sequence of functions $w_{n}$ on $\Delta$ as follows:

$$
w_{n}= \begin{cases}u_{k} & \text { on } U_{k}, k=1, \cdots, n, \\ u_{k}^{\prime} & \text { on } U_{k}^{\prime}, k=1, \cdots, n, \\ 0 & \text { elsewhere in } \Delta .\end{cases}
$$

Each function $w_{n}$ is subharmonic on $\Delta$. To see this observe that the $u_{k}$ and $u_{k}^{\prime}$ vanish on the top and bottom edges of their domains and are positive near these edges. Hence properties (ii) and (iii) surely hold along the edges of the appropriate $U_{k}$ and $U_{k}^{\prime}$. Elsewhere $w_{n}$ is either locally constant $\left(w_{n} \equiv 0\right)$ or else locally agrees with one of the subharmonic functions $u_{k}$ or $u_{k}^{\prime}(k \leq n)$.

The sequence $w_{n}$ converges uniformly to a function $w$ defined on $\Delta$. Because each $w_{n}$ is continuous and the convergence is uniform, $w$ itself is continuous on $\Delta$. Also $w$ satisfies the mean value inequality, property (iii), since the convergence is uniform and all the $w_{n}$ and $w$ are Riemann integrable on any circle contained in $\Delta$. To summarize, the function $w$ is bounded, continuous and subharmonic on $\Delta$.

Clearly $w$ vanishes on $S$ and on the two "flaps" of $\Delta$ given by $|x| \geq 3 / 4$, while in each $U_{n}$ and $U_{n}^{\prime}$ there is a trough where $w$ takes on negative values. 
Thus, $C\left(z_{0}, 0\right)=S$; the two vertical boundary segments of the closure of $S$ are not in $C\left(z_{0}, 0\right)$, because, by construction, a continuum in $\Delta$ which contains $z_{0}$ and meets either segment would have to cut across infinitely many troughs' and therefore could not lie in $C\left(z_{0}, 0\right)$.

\section{BIBLIOGRAPHY}

1. M. Brelot, Étude des fonctions sous-harmoniques au voisinage d'un point singulier, Ann. Inst. Fourier Grenoble 1 (1949), 121-156. MR 12, 258.

2. W. K. Hayman, On the characteristic of functions meromorphic in the unit disk and of their integrals, Acta Math. 112 (1964), 181-214. MR 29 \#6019.

3. Maurice Heins, Seclected topics in the classical theory of functions of a complex variable, Athena Series: Selected Topics in Math., Holt, Rinehart and Winston, New York, 1962. MR $29 \# 217$.

4. J. G. Hocking and G. S. Young, Topology, Addison-Wesley, Reading, Mass., 1961. MR 23 \#A2857.

5. R. Hornblower, Subharmonic analogues of MacLane's classes, Ann. Polon. Math. (to appear).

6. G. R. MacLane, Asymptotic values of holomorphic functions, Rice Univ. Studies 49 (1963), no. 1,83 pp. MR 26 \#6419.

7. M. N. M. Talpur, Thesis, London, 1967.

DEPARTMENT OF MATHEMATICS, SUNY AT ALBANY, ALBANY, NEW YORK 12203 